\title{
MEASUREMENT OF TRANSIENT THERMAL RESPONSE OF MICRO-MIRRORS
}

\author{
Craig R. Forest ${ }^{1}$ \\ Mechanical Engineering Department, Massachusetts Institute of Technology \\ Cambridge, MA 02139
}

P. Reynolds-Browne, J. Harris, E. Novak

Veeco Instruments, Inc.

Tucson, AZ 85706-7123

\section{ABSTRACT}

Metrology of MEMS can provide feedback for modeling of thermal-mechanical behavior and understanding thin film properties. Towards this end, measurements of the transient thermal response of a $500 \mu \mathrm{m}$ diameter polysilicon mirror were performed, as heated with a $100 \mu \mathrm{m}$ diameter infrared laser at optical powers up to $600 \mathrm{~mW}$. Stroboscopic interferometry and thermography were utilized to measure mirror surface topography during heating/cooling and correlate the measured deformation with surface temperature. Transient thermal response occured over approximately $2.2 \mathrm{~ms}$, as measured with $0.14 \mathrm{~ms}$ temporal resolution. Surface temperatures rose to $179^{\circ} \mathrm{C}$ with a corresponding surface deformation amplitude of $124 \mathrm{~nm}$ near the threshold for plastic deformation. Deformation amplitude and surface temperature were proportional to optical power.

\section{INTRODUCTION}

Metrology of MEMS is vital to their widespread application in that it provides feedback for design and manufacturing process improvement which leads to predictable, reliable performance. Since MEMS usually contain moving parts, characterization of dynamic behavior is also useful. Metrology at MEMS length and time scales can lead to new understanding of thin film heat transfer properties for better modeling.

\subsection{MICRO-SWITCHING SYSTEM}

A two-position switch is being developed at Sandia National Laboratories for surety applications [1]. The system features a gear-shaped mirror, a vertical cavity surface emitting laser and a photo-detector. The mirror, shown in Fig. 1, has an off-axis thru-hole; its angular position is controlled by an electrostatic comb drive.

In the design, incident radiation from a laser either passes directly through the off-axis hole and a via in the substrate, or the mirror can be rotated to reflect this radiation. In the latter configuration, radiation incident

\footnotetext{
${ }^{1}$ Travel support has been generously provided by the Transducers Research Foundation and by the DARPA MEMS and DARPA BioFlips programs.

${ }^{2}$ Sandia is a multiprogram laboratory operated by Sandia Corp., a Lockheed Martin Company, for the U.S. Dept. of Energy's Natl. Nuclear Sec. Admin. under Contract DE-AC04-94AL85000.
}

O. Blum-Spahn, C.C. Wong, S. Mani, F. Peter, D. Adams Sandia National Laboratories ${ }^{2}$ Albuquerque, NM 87185

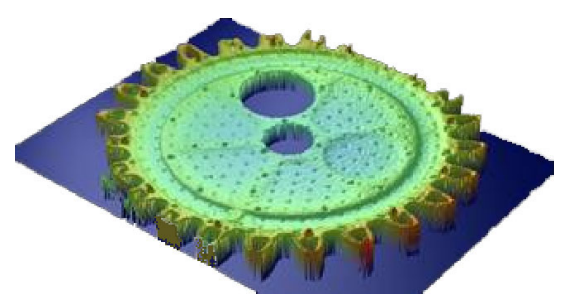

Figure 1: Polysilicon micro-mirror, $500 \mu m$ diameter.

on the mirror is also partially absorbed, causing localized spot heating. Consequent thermal expansion and deformation create undesirable mechanical and optical performance, such as spurious reflections or binding between the mirror and its hub, which can prevent rotation.

Understanding the heat transfer in this system is crucial to mitigating performance concerns. A thermomechanical model in which absorbed radiation results in predictable deformation can be used to redesign the switch to prevent binding by minimizing or moving deformation to an area where there is no performance impact [2]. Empirical data is required to generate this model, so we have pursued metrology of the thermallyinduced mirror deformation as a function of time, incident optical powers, and surface temperatures.

\section{EXPERIMENTAL APPARATUS}

To induce thermal deformation, a laser operating at $832 \mathrm{~nm}$ was connected to an optical fiber. The $100 \mu \mathrm{m}$ diameter bare fiber core was then positioned over the micro-mirror at $45^{\circ}$ incidence using a translation stage, thereby replicating the configuration for the actual optical switch in which local spot heating occurs.

\subsection{DEFORMATION MEASUREMENT}

A stroboscopic interferometer (Veeco Instruments, Inc., NT 1100) [3] was modified to capture the mirror thermal deformations as they occurred. A schematic of the experimental setup is shown in Fig. 2 [4]. In the technique, pulse square-wave signals drive the laser, CCD and LED at the same frequency. The phase of the laser heating, $\phi_{1}$, was varied constantly with respect to the phase of the image capture (CCD and LED), $\phi_{2}$. This resulted in a set of data which fully recorded the mirror heating/cooling cycle. Signals at $20 \mathrm{~Hz}$ and $50 \%$ duty cycle were used. The phase shift, $\Delta \phi=\phi_{1}-\phi_{2}$, was initially zero and was incremented by $1^{\circ} /$ cycle. 


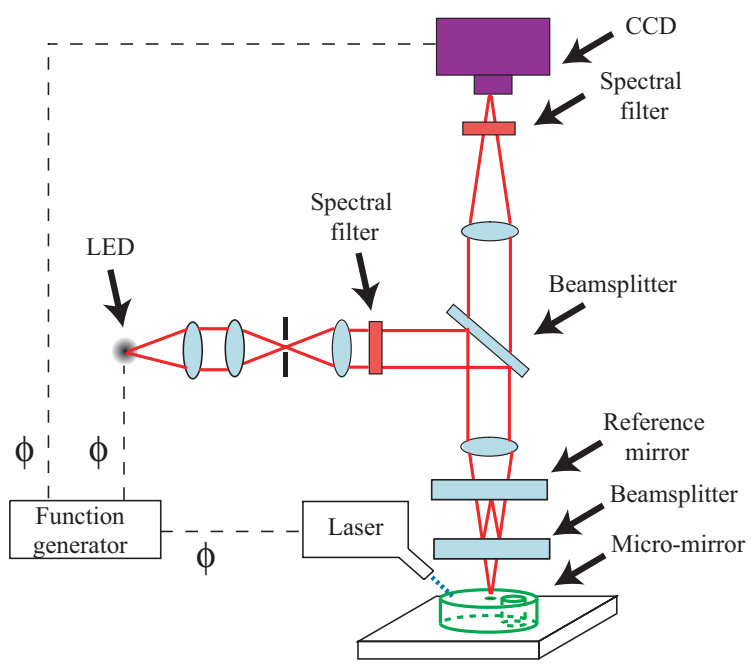

Figure 2. Stroboscopic interferometry apparatus for transient thermal deformation measurements.

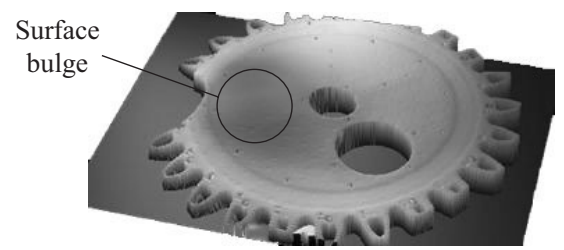

Figure 3. Deformed mirror-compare with Fig. 1. Data absence at mirror's left edge caused by fiber obstruction.

For data integrity, the mirror heating/cooling cycle must be repeatable, or purely elastic. To determine the threshold for plastic deformations, mirrors were subjected to constant laser powers up to $600 \mathrm{~mW}$, where local melting was observed. Based on these results, stroboscopic interferometry experiments were performed below the melting threshold, at $150 \mathrm{~mW}$ and $300 \mathrm{~mW}$.

\subsection{TEMPERATURE MEASUREMENT}

Temperatures on the mirror surface were measured with an infrared microscope (EDO Corporation, Infrascope) with $5 \mu \mathrm{m}$ and $0.1^{\circ} \mathrm{C}$ spatial and temperature resolutions, respectively. Emissivity was calibrated at 50 and $75^{\circ} \mathrm{C}$. Surface temperature measurements were made while the mirror was at steady-state for a range of incident optical powers in the elastic deformation regime.

\section{RESULTS AND DISCUSSION}

The power threshold for plastic deformation was consistently $400 \mathrm{~mW}$. An interferometric image of a micromirror deformed elastically due to local thermal expansion is shown in Fig. 3. By comparing the bulge peakvalley $(\mathrm{P}-\mathrm{V})$ vs. optical power and temperature vs. optical power, we computed the linear relationship between bulge $\mathrm{P}-\mathrm{V}$ and temperature, shown in Fig. 4.

Transient micro-mirror heating/cooling occurs over $2.2 \mathrm{~ms}$, as measured with a $0.14 \mathrm{~ms}$ temporal resolution using stroboscopic interferometry. Results are shown in Fig. 5. Without this stroboscopic modification, interferometry can not measure such short-lived deformations.

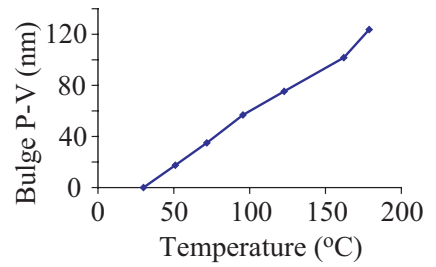

Figure 4. Bulge P-V vs. surface temperature, revealed by comparing interferometry and thermography results.

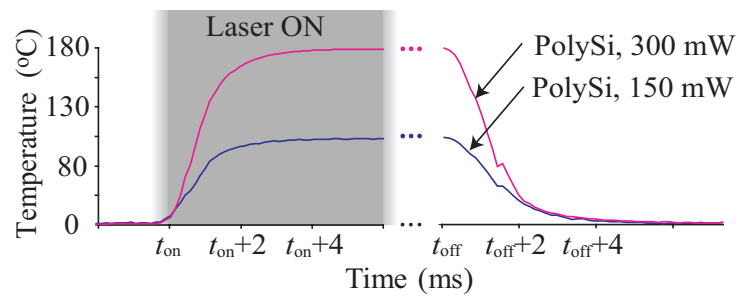

Figure 5: Interferometric heating/cooling results.

Incorporation of thermography measurements permits temperature to deformation correlation. Temperatures of the mirror surface ranged up to $179^{\circ} \mathrm{C}$ at a bulge P-V of $124 \mathrm{~nm}$ due to $300 \mathrm{~mW}$ of incident radiation. Results indicate that for the $<400 \mathrm{~mW}$ elastic regime, deformation is proportional to optical power.

\section{CONCLUSIONS}

The micro-mirror thermal-mechanical behavior has been characterized using a novel application stroboscopic interferometry and infrared thermography. Issues such as how the heat can be better distributed and how deformation can be reduced in critical locations such as the bearing hub can be better understood and solved.

These observations of repeatable, transient MEMS thermal response provide an essential contribution to understanding heat transfer in thin films. The correlation between surface temperature and deformation permits the quantification of radiant and conductive energy rates, which are essential for thermal modeling. Multiphysics analysis software can be validated and improved by comparison with these experimental results. There are numerous other applications in which such dynamic measurement of the transient thermal response of MEMS can be useful, such as bolometers, thermal actuators, and temperature sensitive safety mechanisms.

\section{REFERENCES}

1. A.D. Oliver et al., "Microsystems based on surface micromachined mechanisms", Proceedings of IEEE, Sensors, 2, pp. 1188-1192 (2002).

2. C.C. Wong and S. Graham, "Investigating the thermal response of a micro-optical shutter", IEEE Transactions on Components and Packaging Technologies, 26, 2 (2003).

3. E. Novak et al., "MEMS metrology using a strobed interferometric system", ASPE Winter Topical Mtg. (2003).

4. C.R. Forest et al., "Static and dynamic optical metrology of micro-mirror thermal deformation", NSTI Nanotech Conference (2004). 